\title{
TAIL DEPENDENCE FOR HEAVY-TAILED SCALE MIXTURES OF MULTIVARIATE DISTRIBUTIONS
}

\author{
HAIJUN LI*** AND \\ YANNAN SUN, ${ }^{* * * *}$ Washington State University
}

\begin{abstract}
The tail dependence of multivariate distributions is frequently studied via the tool of copulas. In this paper we develop a general method, which is based on multivariate regular variation, to evaluate the tail dependence of heavy-tailed scale mixtures of multivariate distributions, whose copulas are not explicitly accessible. Tractable formulae for tail dependence parameters are derived, and a sufficient condition under which the parameters are monotone with respect to the heavy tail index is obtained. The multivariate elliptical distributions are discussed to illustrate the results.

Keywords: Tail dependence; heavy tail; multivariate regular variation; copula; hazard rate ordering; multivariate elliptical distribution
\end{abstract}

2000 Mathematics Subject Classification: Primary 62H20; 62P05

\section{Introduction}

Tail dependence parameters describe the amount of dependence in the upper tail or lower tail of a multivariate distribution and can be used to analyze the dependence among extreme values. This paper focuses on the tail dependence of multivariate distributions of random vectors of the form

$$
\left(X_{1}, \ldots, X_{d}\right):=\left(R T_{1}, \ldots, R T_{d}\right),
$$

where $\left(T_{1}, \ldots, T_{d}\right)$ has the joint distribution $G\left(t_{1}, \ldots, t_{d}\right)$ with some finite moments, and the scale variable $R$, independent of $\left(T_{1}, \ldots, T_{d}\right)$, has a regularly varying right tail at infinity with survival function (see, e.g. [3, pp. 17-21] for details),

$$
\bar{F}(r):=1-F(r)=\frac{L(r)}{r^{\alpha}}, \quad r>0, \alpha>0,
$$

where $L$ is a slowly varying function; that is, $L$ is a positive function on $(0, \infty)$ with the property that

$$
\lim _{r \rightarrow \infty} \frac{L(c r)}{L(r)}=1 \quad \text { for every } c>0 .
$$

The class of distributions of the form (1.1) has a variety of interpretations in different applications, including, for example, multivariate elliptical distributions and various multivariate Pareto distributions as special cases.

It is possible to define and analyze tail dependence via copulas; however, the copula method's effectiveness diminishes in higher dimensions and in the case of (1.1), where the copulas are

Received 8 March 2007; revision received 14 September 2009.

* Postal address: Department of Mathematics, Washington State University, Pullman, WA 99164, USA.

** Email address: lih@math.wsu.edu

Research supported in part by NSF grant CMMI 0825960.

*** Email address: ysun@math.wsu.edu 
often not explicitly accessible. A copula $C$ is a distribution function, defined on the unit cube $[0,1]^{d}$, with uniform one-dimensional margins. Given a copula $C$, if we define

$$
F\left(t_{1}, \ldots, t_{d}\right):=C\left(F_{1}\left(t_{1}\right), \ldots, F_{d}\left(t_{d}\right)\right), \quad\left(t_{1}, \ldots, t_{d}\right) \in \mathbb{R}^{d},
$$

then $F$ is a multivariate distribution with univariate margins $F_{1}, \ldots, F_{d}$. Given a distribution $F$ with margins $F_{1}, \ldots, F_{d}$, there exists a copula $C$ such that (1.4) holds. If $F_{1}, \ldots, F_{d}$ are all continuous then the corresponding copula $C$ is unique and can be written as

$$
C\left(u_{1}, \ldots, u_{d}\right)=F\left(F_{1}^{-1}\left(u_{1}\right), \ldots, F_{d}^{-1}\left(u_{d}\right)\right), \quad\left(u_{1}, \ldots, u_{d}\right) \in[0,1]^{d} .
$$

Thus, for multivariate distributions with continuous margins, the univariate margins and the multivariate dependence structure (as described by their copulas) can be separated [17], [23].

The survival copula is defined similarly. Consider a random vector $\left(X_{1}, \ldots, X_{d}\right)$ with continuous margins $F_{1}, \ldots, F_{d}$ and copula $C$. Observe that $\bar{F}_{i}\left(X_{i}\right)=1-F_{i}\left(X_{i}\right), 1 \leq i \leq d$, is also uniformly distributed over $[0,1]$; thus

$$
\hat{C}\left(u_{1}, \ldots, u_{d}\right):=\operatorname{Pr}\left\{\bar{F}_{1}\left(X_{1}\right) \leq u_{1}, \ldots, \bar{F}_{d}\left(X_{d}\right) \leq u_{d}\right\}
$$

is a copula. We call $\hat{C}$ the survival copula of $\left(X_{1}, \ldots, X_{d}\right)$. The joint survival function of the random vector $\left(X_{1}, \ldots, X_{d}\right)$ is expressed as

$$
\bar{F}\left(t_{1}, \ldots, t_{d}\right):=\operatorname{Pr}\left\{X_{1}>t_{1}, \ldots, X_{d}>t_{d}\right\}=\hat{C}\left(\bar{F}_{1}\left(t_{1}\right), \ldots, \bar{F}_{d}\left(t_{d}\right)\right), \quad\left(t_{1}, \ldots, t_{d}\right) \in \mathbb{R}^{d} .
$$

It also follows that, for any $\left(u_{1}, \ldots, u_{d}\right) \in[0,1]^{d}$,

$$
\bar{C}\left(u_{1}, \ldots, u_{d}\right):=\operatorname{Pr}\left\{F_{1}\left(X_{1}\right)>u_{1}, \ldots, F_{d}\left(X_{d}\right)>u_{d}\right\}=\hat{C}\left(1-u_{1}, \ldots, 1-u_{d}\right),
$$

where $\bar{C}$ is the joint survival function of copula $C$.

The bivariate tail dependence parameter has been discussed extensively in [11]. Moreover, various multivariate tail dependence parameters can be introduced. Definition 1.1, below, details two notions of tail dependence which have been discussed in [14] and [21].

Definition 1.1. Let $\boldsymbol{X}=\left(X_{1}, \ldots, X_{d}\right)$ be a random vector with continuous margins $F_{1}, \ldots, F_{d}$ and copula $C$. Let $N$ denote the index set $\{1, \ldots, d\}$.

1. $\boldsymbol{X}$ is said to be upper-orthant tail dependent if, for some subset $\varnothing \neq J \subset N$, the following limit exists and is positive:

$$
\tau_{J}=\lim _{u \rightarrow 1^{-}} \operatorname{Pr}\left\{F_{j}\left(X_{j}\right)>u \text { for all } j \in N \backslash J \mid F_{i}\left(X_{i}\right)>u \text { for all } i \in J\right\}>0 .
$$

If, for all $\varnothing \neq J \subset N, \tau_{J}=0$, then we say that $\boldsymbol{X}$ is upper-orthant tail independent.

2. $\boldsymbol{X}$ is said to be upper extremal dependent if the following limit exists and is positive:

$$
\gamma=\lim _{u \rightarrow 1^{-}} \operatorname{Pr}\left\{F_{j}\left(X_{j}\right)>u \text { for all } j \in N \mid F_{i}\left(X_{i}\right)>u \text { there exists } i \in N\right\}>0 .
$$

If $\gamma=0$ then we say that $\boldsymbol{X}$ is upper extremal independent.

The lower tail dependence of a copula is defined as the upper tail dependence of its survival copula in (1.5). The limits $\tau_{J}$ and $\gamma$ are called the upper tail and extremal dependence parameters, respectively. Obviously, tail dependence is a copula property; these parameters do 
not depend on the marginal distributions. If $X_{1}, \ldots, X_{n}$ are independent then the corresponding upper tail (extremal) dependence parameters are all zeros. Clearly, $\tau_{J} \geq \gamma$ for all nonempty $J \subset N$. Thus, the extremal dependence parameter provides a lower bound for orthant tail dependence parameters. The multivariate tail dependence parameters, the $\tau_{J} \mathrm{~s}$, have been used in [9] to analyze the contagion risk in banking systems, and the extremal dependence parameter $\gamma$ in the bivariate case has been used in [4] to analyze the extremal dependence in financial return data.

Definition 1.1 implies that the tail dependence parameters of a distribution can be derived directly from its copula, and this has been done for the bivariate case [5], [11]. In [14] and [15], using the copula method, Li derived explicit expressions of the orthant tail dependence for Marshall-Olkin distributions as well as multivariate extreme value distributions and their scaled mixtures. It is already evident in [14] that even if the copula is explicitly available, the direct copula method has to equalize the distribution margins by taking componentwise marginal transforms (see (1.4)); this method becomes really cumbersome in higher dimensions. The copula method is obviously ineffective for (1.1), whose copulas are generally not explicitly accessible. This paper develops an alternative method to derive tractable tail dependence formulae for (1.1), which belong to the family of multivariate regularly varying distributions [1], [2]. Our method is rooted in the theory of multivariate regular variation [19], [20], and, thus, unlike the copula method, our method avoids taking marginal transforms on the entire distribution. It is well known that the bivariate normal distribution is asymptotically tail independent if its correlation coefficient $\rho$ is less than 1. Bivariate elliptical distributions, a special case of (1.1), possess the upper- (and lower-) orthant tail dependence property if their generating random variable is regularly varying [10], [21]. The formula for the extremal dependence $\gamma$ of bivariate elliptical distributions is also derived in [8]. Our method not only yields similar results for multivariate elliptical distributions, but also captures a more detailed relationship between various tail/extremal dependence parameters and the theory of multivariate regular variation.

The paper is organized as follows. In Section 2 we first discuss the tail dependence functions, which can be used to express various tail dependence parameters. Furthermore, it is shown that, for a multivariate regularly varying distribution, the upper tail dependence function and the intensity measure are equivalent in describing its extremal dependence structure. In Section 3 we derive explicit expressions for the tail dependence of (1.1). Multivariate elliptical distributions are also discussed as examples. Finally, some comments in Section 4 conclude the paper. Throughout this paper, the terms 'increasing' and 'decreasing' are used in the weak sense, and the measurability of functions is assumed. The notation $a \vee b$ and $a \wedge b$ mean the maximum and the minimum of $a$ and $b$, respectively.

\section{Tail dependence function of multivariate regular variation}

Let $F$ be the distribution function (DF) of a $d$-dimensional random vector $\boldsymbol{X}=\left(X_{1}, \ldots, X_{d}\right)$ with continuous margins $F_{1}, \ldots, F_{d}$ and copula $C$. The lower and upper tail dependence functions, denoted by $b(\cdot ; C)$ and $b^{*}(\cdot ; C)$, respectively, are introduced in [12], [13], and [18] as follows:

$$
\begin{aligned}
b(\boldsymbol{w} ; C):=\lim _{u \rightarrow 0^{+}} \frac{C\left(u w_{j} \text { for all } j \in N\right)}{u} & \text { for all } \boldsymbol{w}=\left(w_{1}, \ldots, w_{d}\right) \in \mathbb{R}_{+}^{d}, \\
b^{*}(\boldsymbol{w} ; C):=\lim _{u \rightarrow 0^{+}} \frac{\bar{C}\left(1-u w_{j} \text { for all } j \in N\right)}{u} & \text { for all } \boldsymbol{w}=\left(w_{1}, \ldots, w_{d}\right) \in \mathbb{R}_{+}^{d},
\end{aligned}
$$


provided that the limits exist. Since $b(\boldsymbol{w} ; \hat{C})=b^{*}(\boldsymbol{w} ; C)$, where $\hat{C}\left(u_{1}, \ldots, u_{d}\right)=\bar{C}(1-$ $\left.u_{1}, \ldots, 1-u_{d}\right)$ is the survival copula in (1.5), this paper focuses only on the upper tail dependence. The explicit expression of $b^{*}$ for elliptical distributions was obtained in [13]. A theory of tail dependence functions was developed in [12] and [18], based on Euler's homogeneous representation:

$$
b^{*}(\boldsymbol{w} ; C)=\sum_{j=1}^{d} w_{j} t_{j}\left(w_{i}, i \neq j \mid w_{j}\right) \quad \text { for all } \boldsymbol{w}=\left(w_{1}, \ldots, w_{d}\right) \in \mathbb{R}_{+}^{d},
$$

where

$$
\begin{aligned}
& t_{j}\left(w_{i}, i \neq j \mid w_{j}\right) \\
& \quad:=\lim _{u \rightarrow 0^{+}} \operatorname{Pr}\left\{F_{i}\left(X_{i}\right)>1-w_{i} u \text { for all } i \neq j \mid F_{j}\left(X_{j}\right)=1-w_{j} u\right\}, \quad j \in N .
\end{aligned}
$$

The $t_{j} \mathrm{~s}$ are called upper conditional tail dependence functions. For copulas with explicit expressions, the tail dependence functions are obtained directly from the copulas with relative ease. For copulas without explicit expressions, the tail dependence functions can be obtained from (2.2) by exploring closure properties of the related conditional distributions. In [18], for example, the tail dependence function of the multivariate $t$ distribution is obtained by (2.2).

It follows from (1.6) and (2.1) that the upper tail dependence parameters can be expressed as

$$
\tau_{J}=\frac{b^{*}(1, \ldots, 1 ; C)}{b^{*}\left(1, \ldots, 1 ; C_{J}\right)} \quad \text { for all } \varnothing \neq J \subset N,
$$

where $C_{J}$ is the multivariate margin of $C$ with component indexes in $J$. It was shown in [12] that $b^{*}(\boldsymbol{w} ; C)>0$ for all $\boldsymbol{w} \in \mathbb{R}_{+}^{d}$ if and only if $b^{*}(1, \ldots, 1 ; C)>0$. Unlike the $\tau_{J}$, however, the tail dependence function provides all the extremal dependence information of the copula $C$ as specified by its extreme value copula (EV copula). The upper EV copula of $C$, denoted by $C^{\mathrm{UEV}}$, is defined as $C^{\mathrm{UEV}}\left(u_{1}, \ldots, u_{d}\right):=\lim _{n \rightarrow \infty} C^{n}\left(u_{1}^{1 / n}, \ldots, u_{d}^{1 / n}\right)$ for any $\left(u_{1}, \ldots, u_{d}\right) \in[0,1]^{d}$ if the limit exists $[11]$.

Define the upper exponent function $a^{*}(\cdot ; C)$ of copula $C$ as

$$
a^{*}(\boldsymbol{w} ; C):=\sum_{\substack{S \subseteq N \\ S \neq \varnothing}}(-1)^{|S|-1} b_{S}^{*}\left(w_{i}, i \in S ; C_{S}\right),
$$

where $b_{S}^{*}\left(w_{i}, i \in S ; C_{S}\right)$ denotes the upper tail dependence function of the margin $C_{S}$ of $C$ with component indexes in $S$. It follows from (1.7) and (2.4) that the extremal dependence parameter can be expressed as

$$
\gamma=\frac{b^{*}(1, \ldots, 1 ; C)}{a^{*}(1, \ldots, 1 ; C)}
$$

Similar to tail dependence functions, the exponent function has the following homogeneous representation:

$$
a^{*}(\boldsymbol{w} ; C)=\sum_{j=1}^{d} w_{j} \bar{t}_{j}\left(w_{i}, i \neq j \mid w_{j}\right) \quad \text { for all } \boldsymbol{w}=\left(w_{1}, \ldots, w_{d}\right) \in \mathbb{R}_{+}^{d},
$$


where

$$
\begin{aligned}
& \bar{t}_{j}\left(w_{i}, i\right.\left.\neq j \mid w_{j}\right) \\
& \quad=\lim _{u \rightarrow 0^{+}} \operatorname{Pr}\left\{F_{i}\left(X_{i}\right) \leq 1-w_{i} u \text { for all } i \neq j \mid F_{j}\left(X_{j}\right)=1-w_{j} u\right\}, \quad j \in N .
\end{aligned}
$$

It was shown in [12] that tail dependence functions $\left\{b_{S}^{*}\left(w_{i}, i \in S ; C_{S}\right)\right\}$ and the exponent function $a^{*}(\boldsymbol{w} ; C)$ are uniquely determined from each other.

Theorem 2.1. ([12] and [18].) Let C be a d-dimensional copula. Then the upper EV copula is defined as

$$
C^{\mathrm{UEV}}\left(u_{1}, \ldots, u_{d}\right)=\exp \left\{-a^{*}\left(-\log u_{1}, \ldots,-\log u_{d}\right)\right\} \text { for all }\left(u_{1}, \ldots, u_{d}\right) \in[0,1]^{d},
$$

where $a^{*}$ is given by either (2.4) or (2.6).

Using the language of extreme value theory (see, e.g. Chapter 7 of [16]), if the upper tail dependence function $b^{*}(\cdot ; C)$ exists then the copula $C$ is in the copula domain of attraction of the EV copula $C^{\mathrm{UEV}}$, and $C^{\mathrm{UEV}}$ is uniquely determined by $b^{*}(\cdot ; C)$. Next, we will show that the upper tail dependence function and the intensity measure for multivariate regular variation are also uniquely determined from each other. For notational convenience, we denote hereafter by $[\boldsymbol{a}, \boldsymbol{b}]$ the Cartesian product $\prod_{i=1}^{d}\left[a_{i}, b_{i}\right]$, where $\boldsymbol{a}, \boldsymbol{b} \in \overline{\mathbb{R}}^{d}$ and $a_{i} \leq b_{i}$ for each $i$. The following definition can be found in [7, pp. 185-286], [19], and [20].

Definition 2.1. A random vector $\boldsymbol{X}$ is said to be multivariate regularly varying (MRV) if there exists a Radon measure $\mu$ (i.e. finite on compact sets), called the intensity measure, on $\overline{\mathbb{R}}^{d} \backslash\{\mathbf{0}\}$, where $\mathbf{0}$ is the origin in $\overline{\mathbb{R}}^{d}$, such that

$$
\lim _{t \rightarrow \infty} \frac{\operatorname{Pr}\{\boldsymbol{X} \in t B\}}{\operatorname{Pr}\{\|\boldsymbol{X}\|>t\}}=\mu(B)
$$

for any relatively compact set $B \subset \overline{\mathbb{R}}^{d} \backslash\{\mathbf{0}\}$ that satisfies $\mu(\partial B)=0$, where $\|\cdot\|$ denotes a norm on $\mathbb{R}^{d}$. (Here $\overline{\mathbb{R}}^{d}=[-\infty, \infty]^{d}$ is compact and the punctured version $\overline{\mathbb{R}}^{d} \backslash\{\mathbf{0}\}$ is modified via one-point uncompactification (see, e.g. [20, pp. 168-170]).)

If $\boldsymbol{X}$ is MRV with $\mu\left([\mathbf{0}, \mathbf{1}]^{c}\right)>0$, where $\mathbf{1}$ denotes the vector of $1 \mathrm{~s}$ and $[\mathbf{0}, \mathbf{1}]^{c}$ denotes the complement of $[\mathbf{0}, \mathbf{1}]$ in $\mathbb{R}^{d}$, then, for every relatively compact set $B \subset \overline{\mathbb{R}} \backslash\{\{\mathbf{0}\}$ that satisfies $\mu(\partial B)=0$,

$$
\lim _{t \rightarrow \infty} \frac{\operatorname{Pr}\{\boldsymbol{X} \in t B\}}{\operatorname{Pr}\left\{\boldsymbol{X} \in t[\mathbf{0}, \mathbf{1}]^{c}\right\}}=\tilde{\mu}(B),
$$

where $\tilde{\mu}(B)=\mu(B) / \mu\left([\mathbf{0}, \mathbf{1}]^{c}\right)$. In particular, any MRV DF with support in $\mathbb{R}_{+}^{d}=[0, \infty]^{d}$ admits the following spectral representation.

Theorem 2.2. ([7], [19], and [20].) If $\boldsymbol{X}$ is nonnegative with MRV distribution $F$ then there exists a Radon measure $\tilde{\mu}$ on $\mathbb{R}_{+}^{d}$ such that

$$
\lim _{t \rightarrow \infty} \frac{1-F(t \boldsymbol{x})}{1-F(t \mathbf{1})}=\lim _{t \rightarrow \infty} \frac{\operatorname{Pr}\left\{\boldsymbol{X} / t \in[\mathbf{0}, \boldsymbol{x}]^{c}\right\}}{\operatorname{Pr}\left\{\boldsymbol{X} / t \in[\mathbf{0}, \mathbf{1}]^{c}\right\}}=\tilde{\mu}\left([\mathbf{0}, \boldsymbol{x}]^{c}\right)
$$

for all continuous points $\boldsymbol{x}$ of $\tilde{\mu}$, where $\tilde{\mu}\left([\mathbf{0}, \boldsymbol{x}]^{c}\right)=c \int_{\mathbb{S}_{+}^{d-1}} \max _{1 \leq j \leq d}\left(u_{j} / x_{j}\right)^{\alpha} S(\mathrm{~d} \boldsymbol{u})$ for some constant $c$ and a probability measure $S$ on $\mathbb{S}_{+}^{d-1}:=\left\{\boldsymbol{x} \in \mathbb{R}_{+}^{d}:\|\boldsymbol{x}\|=1\right\}$. 
There is a well-known relation (see Proposition 5.15 of [19] for details) between MRV distributions and multivariate extreme value (MEV) distributions with identical Fréchet margins $H(x ; 1):=\exp \left\{-x^{-1}\right\}$ for $x>0$. In general, the margins of an MEV distribution are expressed in terms of the generalized extreme value family,

$$
H(x ; \gamma):=\exp \left\{-(\max \{1+\gamma x, 0\})^{-1 / \gamma}\right\}, \quad x \in \mathbb{R}, \gamma \in \mathbb{R} .
$$

Note, however, that the parametric feature enjoyed by the univariate EV distributions vanishes in the multivariate context.

Theorem 2.3. Consider a nonnegative random vector $\boldsymbol{X}=\left(X_{1}, \ldots, X_{d}\right)$ with MRV DF F and continuous margins $F_{1}, \ldots, F_{d}$. Let $C^{F}$ and $\mu$ respectively denote the copula and the intensity measure of $F$. If the margins are tail equivalent (i.e. $\bar{F}_{i}(x) / \bar{F}_{j}(x) \rightarrow 1$ as $x \rightarrow \infty$ for any $i \neq j)$ then the upper tail dependence function $b^{*}\left(\cdot ; C^{F}\right)$ exists and

1. $b^{*}\left(\boldsymbol{w} ; C^{F}\right)=\frac{\mu\left(\prod_{i=1}^{d}\left[w_{i}^{-1 / \alpha}, \infty\right]\right)}{\mu\left([1, \infty] \times \overline{\mathbb{R}}_{+}^{d-1}\right)}, \quad a^{*}\left(\boldsymbol{w} ; C^{F}\right)=\frac{\mu\left(\left(\prod_{i=1}^{d}\left[0, w_{i}^{-1 / \alpha}\right]\right)^{c}\right)}{\mu\left(\left([0,1] \times \overline{\mathbb{R}}_{+}^{d-1}\right)^{c}\right)}$,

2. $\frac{\mu([\boldsymbol{w}, \infty])}{\mu\left([\mathbf{0}, \mathbf{1}]^{c}\right)}=\frac{b^{*}\left(\left(w_{1}^{-\alpha}, \ldots, w_{d}^{-\alpha}\right) ; C^{F}\right)}{a^{*}\left((1, \ldots, 1) ; C^{F}\right)}, \quad \frac{\mu\left([\mathbf{0}, \boldsymbol{w}]^{c}\right)}{\mu\left([\mathbf{0}, \mathbf{1}]^{c}\right)}=\frac{a^{*}\left(\left(w_{1}^{-\alpha}, \ldots, w_{d}^{-\alpha}\right) ; C^{F}\right)}{a^{*}\left((1, \ldots, 1) ; C^{F}\right)}$.

Proof. Since each $F_{i}, i \in N$, is regularly varying, from (1.2) and (1.3), we have $\bar{F}_{i}(x)=$ $L_{i}(x) / x^{\alpha}$ for $x \geq 0$. To estimate $\bar{F}_{i}^{-1}\left(w_{i} u\right)$ when $u \rightarrow 0^{+}$for fixed $w_{i}>0$, consider

$$
\bar{F}_{i}\left(w_{i}^{1 / \alpha} x\right)=\frac{L_{i}\left(w_{i}^{1 / \alpha} x\right)}{w_{i} x^{\alpha}}=w_{i}^{-1} \bar{F}_{i}(x) g_{i}\left(w_{i}, x\right)
$$

where $g_{i}\left(w_{i}, x\right):=L_{i}\left(w_{i}^{1 / \alpha} x\right) / L_{i}(x) \rightarrow 1$ as $x \rightarrow \infty$. Substituting $\bar{F}_{i}(x)=w_{i} u$ into the above expression and taking $\bar{F}_{i}^{-1}$ on both sides, we obtain

$$
\bar{F}_{i}^{-1}\left(w_{i} u\right)=w_{i}^{-1 / \alpha} \bar{F}_{i}^{-1}\left(u g_{i}\left(w_{i}, \bar{F}_{i}^{-1}\left(w_{i} u\right)\right)\right) .
$$

Asymptotically, $\bar{F}_{i}^{-1}\left(w_{i} u\right) \approx w_{i}^{-1 / \alpha} \bar{F}_{i}^{-1}(u)$ as $u \rightarrow 0^{+}$.

For any fixed $\boldsymbol{w}=\left(w_{1}, \ldots, w_{d}\right)$ with $w_{i}>0, i \in N$, consider

$$
\begin{aligned}
b^{*}\left(\boldsymbol{w} ; C^{F}\right) & =\lim _{u \rightarrow 0^{+}} \frac{\operatorname{Pr}\left\{F_{i}\left(X_{i}\right)>1-w_{i} u \text { for all } i \in N\right\}}{\operatorname{Pr}\left\{F_{1}\left(X_{1}\right)>1-u\right\}} \\
& =\lim _{u \rightarrow 0^{+}} \frac{\operatorname{Pr}\left\{X_{i}>\bar{F}_{i}^{-1}\left(w_{i} u\right) \text { for all } i \in N\right\}}{\operatorname{Pr}\left\{X_{1}>\bar{F}_{1}^{-1}(u)\right\}} \\
& =\lim _{u \rightarrow 0^{+}} \frac{\operatorname{Pr}\left\{X_{i}>w_{i}^{-1 / \alpha} \bar{F}_{i}^{-1}\left(u g_{i}\left(w_{i}, \bar{F}_{i}^{-1}\left(w_{i} u\right)\right)\right) \text { for all } i \in N\right\}}{\operatorname{Pr}\left\{X_{1}>\bar{F}_{1}^{-1}(u)\right\}} .
\end{aligned}
$$

Since $\bar{F}_{i}(x) / \bar{F}_{1}(x) \rightarrow 1$ as $x \rightarrow \infty$, we have from [20, Proposition 2.6] $\bar{F}_{i}^{-1}(u) / \bar{F}_{1}^{-1}(u) \rightarrow 1$ as $u \rightarrow 0^{+}$. For any small $\varepsilon>0$, when $u$ is sufficiently small,

$$
1-\varepsilon<g_{i}\left(w_{i}, \bar{F}_{i}^{-1}\left(w_{i} u\right)\right)<1+\varepsilon \text { for all } i \in N .
$$

Thus, when $u$ is sufficiently small,

$$
\frac{\bar{F}_{i}^{-1}(u(1-\varepsilon))}{\bar{F}_{1}^{-1}(u)} \geq \frac{\bar{F}_{i}^{-1}\left(u g_{i}\left(w_{i}, \bar{F}_{i}^{-1}\left(w_{i} u\right)\right)\right)}{\bar{F}_{1}^{-1}(u)} \geq \frac{\bar{F}_{i}^{-1}(u(1+\varepsilon))}{\bar{F}_{1}^{-1}(u)} .
$$


Since $\bar{F}_{1}^{-1}(u)$ is regularly varying at 0 (see, e.g. Proposition 2.6 of [20]-this result is stated in [20] in terms of increasing regularly varying functions, but is easily verified to be true for decreasing regularly varying functions) or, more precisely, $\bar{F}_{1}^{-1}(u c) / \bar{F}_{1}^{-1}(u) \rightarrow c^{-1 / \alpha}$ as $u \rightarrow 0^{+}$for any $c>0$, then by taking the limits in (2.11) we have

$$
(1-\varepsilon)^{-1 / \alpha} \geq \frac{\bar{F}_{i}^{-1}\left(u g_{i}\left(w_{i}, \bar{F}_{i}^{-1}\left(w_{i} u\right)\right)\right)}{\bar{F}_{1}^{-1}(u)} \geq(1+\varepsilon)^{-1 / \alpha} \quad \text { as } u \rightarrow 0^{+}
$$

for any small $\varepsilon>0$. That is, when $u$ is sufficiently small,

$$
\begin{aligned}
(1-\varepsilon)^{-1 / \alpha} \bar{F}_{1}^{-1}(u) & \\
& \geq \bar{F}_{i}^{-1}\left(u g_{i}\left(w_{i}, \bar{F}_{i}^{-1}\left(w_{i} u\right)\right)\right) \\
& \geq(1+\varepsilon)^{-1 / \alpha} \bar{F}_{1}^{-1}(u) \text { for all } \varepsilon>0, i \in N .
\end{aligned}
$$

Combining these inequalities with (2.10), we have, for any $\varepsilon>0$,

$$
\begin{aligned}
\lim _{u \rightarrow 0^{+}} & \frac{\operatorname{Pr}\left\{X_{i}>w_{i}^{-1 / \alpha}(1-\varepsilon)^{-1 / \alpha} \bar{F}_{1}^{-1}(u) \text { for all } i \in N\right\}}{\operatorname{Pr}\left\{X_{1}>\bar{F}_{1}^{-1}(u)\right\}} \\
& \leq b^{*}\left(\boldsymbol{w} ; C^{F}\right) \\
& \leq \lim _{u \rightarrow 0^{+}} \frac{\operatorname{Pr}\left\{X_{i}>w_{i}^{-1 / \alpha}(1+\varepsilon)^{-1 / \alpha} \bar{F}_{1}^{-1}(u) \text { for all } i \in N\right\}}{\operatorname{Pr}\left\{X_{1}>\bar{F}_{1}^{-1}(u)\right\}},
\end{aligned}
$$

which implies that, after substituting $t=\bar{F}_{1}^{-1}(u)$,

$$
b^{*}\left(\boldsymbol{w} ; C^{F}\right)=\lim _{t \rightarrow \infty} \frac{\operatorname{Pr}\left\{X_{i}>w_{i}^{-1 / \alpha} t, i \in N\right\}}{\operatorname{Pr}\left\{X_{1}>t\right\}} .
$$

Set $A=\prod_{i=1}^{d}\left[w_{i}^{-1 / \alpha}, \infty\right]$ and $B=[1, \infty] \times \overline{\mathbb{R}}_{+}^{d-1}$. Then (2.7) implies that

$$
b^{*}\left(\boldsymbol{w} ; C^{F}\right)=\frac{\mu(A)}{\mu(B)}=\frac{\mu\left(\prod_{i=1}^{d}\left[w_{i}^{-1 / \alpha}, \infty\right]\right)}{\mu\left([1, \infty] \times \overline{\mathbb{R}}_{+}^{d-1}\right)} .
$$

Similar to (2.10), it follows from (2.4) that

$$
\begin{aligned}
a^{*}\left(\boldsymbol{w} ; C^{F}\right) & =\lim _{u \rightarrow 0^{+}} \frac{\operatorname{Pr}\left\{F_{i}\left(X_{i}\right)>1-w_{i} u \text { there exists } i \in N\right\}}{\operatorname{Pr}\left\{F_{1}\left(X_{1}\right)>1-u\right\}} \\
& =\lim _{u \rightarrow 0^{+}} \frac{\operatorname{Pr}\left\{X_{i}>w_{i}^{-1 / \alpha} \bar{F}_{i}^{-1}\left(u g_{i}\left(w_{i}, \bar{F}_{i}^{-1}\left(w_{i} u\right)\right)\right) \text { there exists } i \in N\right\}}{\operatorname{Pr}\left\{X_{1}>\bar{F}_{1}^{-1}(u)\right\}} .
\end{aligned}
$$

Borrowing from the derivation of (2.12), then using (2.7), we have

$$
a^{*}\left(\boldsymbol{w} ; C^{F}\right)=\lim _{t \rightarrow \infty} \frac{\operatorname{Pr}\left\{X_{i}>w_{i}^{-1 / \alpha} t \text { there exists } i \in N\right\}}{\operatorname{Pr}\left\{X_{1}>t\right\}}=\frac{\mu\left(\left(\prod_{i=1}^{d}\left[0, w_{i}^{-1 / \alpha}\right]\right)^{c}\right)}{\mu\left([1, \infty] \times \overline{\mathbb{R}}_{+}^{d-1}\right)} .
$$

The expressions in part 2 can be easily obtained from part 1 . 
Observe that the rescaled intensity measure $\tilde{\mu}(B)=\mu(B) / \mu\left([\mathbf{0}, \mathbf{1}]^{c}\right)$ in part 2 of Theorem 2.3 (also see $(2.8)$ and $(2.9))$ satisfies that $\tilde{\mu}\left(([\mathbf{0}, \mathbf{1}])^{c}\right)=1$. The intensity measures $\mu$ and $\tilde{\mu}$ are uniquely determined from each other.

In general, $\bar{F}_{i}(x) / \bar{F}_{j}(x) \rightarrow r_{i j}$ as $x \rightarrow \infty$ for any $i \neq j$ [20, p. 174]. If $0<r_{i j}<\infty$ then Theorem 2.3 still holds by properly adjusting the marginal scaling constants. If $r_{i j}=0$ or $r_{i j}=\infty$, then some margins have heavier tails than others and more subtle separate marginal scalings are needed to derive the limiting results.

\section{Tail dependence of heavy-tailed scale mixtures of multivariate distributions}

As shown in Theorem 2.3, the upper tail dependence function represents a rescaled version of the intensity measure. The link presented in Theorem 2.3 allows people, on the one hand, to develop, via tail dependence functions, tractable parametric models for the intensity measure, and, on the other hand, to derive, via multivariate regular variation, tail dependence functions in the situations where copulas have neither explicit analytic expressions nor closure properties for their conditional distributions. We illustrate the latter part in this section for the random vectors of the form (1.1).

Let $T_{i+}:=T_{i} \vee 0, i \in N$, and assume in this section that $0<\mathrm{E}\left(T_{i+}^{\alpha+\varepsilon}\right)<\infty$ for some $\varepsilon>0$. Since $R$ is regularly varying with survival function (1.2), then, by Breiman's theorem (see, e.g. Proposition 7.5 of [20]), $R T_{i+}, i \in N$, is also regularly varying with survival function $\operatorname{Pr}\left\{R T_{i+}>r\right\}=\mathrm{E}\left(T_{i+}^{\alpha}\right) L_{i}(r) / r^{\alpha}$, where $L_{i}(r) / L_{j}(r) \rightarrow 1$ as $r \rightarrow \infty$ for $i \neq j$. Consider

$$
\left(Y_{1}, \ldots, Y_{d}\right)^{\top}:=D\left[\left(\mathrm{E}\left(T_{1+}^{\alpha}\right)\right)^{-1 / \alpha} T_{1+}, \ldots,\left(\mathrm{E}\left(T_{d+}^{\alpha}\right)\right)^{-1 / \alpha} T_{d+}\right](R, \ldots, R)^{\top},
$$

where $\boldsymbol{D}\left[a_{1}, \ldots, a_{d}\right]$ is a $d \times d$ diagonal matrix with main diagonal entries $a_{1}, \ldots, a_{d}$ (here and hereafter ' $T$, denotes the matrix transpose). Since $Y_{i}$ is strictly increasing in $X_{i} \geq 0, i \in N$, $\left(Y_{1}, \ldots, Y_{d}\right)$ in (3.1) and $\left(X_{1}, \ldots, X_{d}\right)$ in (1.1) have the same upper tail dependence function. It follows from Proposition A.1 of [2] that $\left(Y_{1}, \ldots, Y_{d}\right)$ is regularly varying, and it is also easy to verify that $\operatorname{Pr}\left\{Y_{i}>r\right\} / \operatorname{Pr}\left\{Y_{j}>r\right\} \rightarrow 1$ as $r \rightarrow \infty$. Thus, by Theorem 2.3, the expression of the upper tail dependence function of $\left(X_{1}, \ldots, X_{d}\right)$ boils down to the determination of the intensity measure of $\left(Y_{1}, \ldots, Y_{d}\right)$ in (3.1).

Proposition A.1 of [2] presents a general formula for the intensity measure of a random affine transform of a random vector that is regularly varying. Applying this formula to (3.1), the intensity measure $\mu$ of (3.1) is given by, for any Borel measurable subset $B \subseteq \overline{\mathbb{R}}_{+}^{d}$,

$$
\mu(B)=\mathrm{E}\left(v\left(D\left[\left(\mathrm{E}\left(T_{1+}^{\alpha}\right)\right)^{1 / \alpha} T_{1+}^{-1}, \ldots,\left(\mathrm{E}\left(T_{d+}^{\alpha}\right)\right)^{1 / \alpha} T_{d+}^{-1}\right](B)\right)\right),
$$

where $v$ is the intensity measure of $(R, \ldots, R)$. Using (2.9), we have, for any nonnegative $\boldsymbol{w}=\left(w_{1}, \ldots, w_{d}\right)$,

$$
v\left([\mathbf{0}, \boldsymbol{w}]^{c}\right)=\lim _{t \rightarrow \infty} \frac{\operatorname{Pr}\left\{R>t \bigwedge_{i=1}^{d} w_{i}\right\}}{\operatorname{Pr}\{R>t\}}=\bigvee_{i=1}^{d} w_{i}^{-\alpha} .
$$

Using the inclusion-exclusion relation and the fact that $\sum_{\varnothing \neq S \subseteq N}(-1)^{|S|-1} \bigvee_{i \in S} w_{i}=\bigwedge_{i \in N} w_{i}$ for all nonnegative $w_{1}, \ldots, w_{d}$, we also have

$$
v([\boldsymbol{w}, \infty])=\bigwedge_{i=1}^{d} w_{i}^{-\alpha}
$$


Substituting these two expressions into (3.2), then

$$
\mu\left([\mathbf{0}, \boldsymbol{w}]^{c}\right)=\mathrm{E}\left(\bigvee_{i=1}^{d} \frac{w_{i}^{-\alpha} T_{i+}^{\alpha}}{\mathrm{E}\left(T_{i+}^{\alpha}\right)}\right) \text { and } \mu([\boldsymbol{w}, \infty])=\mathrm{E}\left(\bigwedge_{i=1}^{d} \frac{w_{i}^{-\alpha} T_{i+}^{\alpha}}{\mathrm{E}\left(T_{i+}^{\alpha}\right)}\right),
$$

which lead to the expression of the upper tail dependence function.

In summary, we give the following theorem.

Theorem 3.1. Consider a random vector $\boldsymbol{X}=\left(R T_{1}, \ldots, R T_{d}\right)$ in the form of (1.1) with $D F F$ and continuous margins $F_{1}, \ldots, F_{d}$. Assume that $0<\mathrm{E}\left(T_{i+}^{\alpha+\varepsilon}\right)<\infty, i \in N$, for some $\varepsilon>0$. Then the upper tail dependence and exponent functions are given by

$$
b^{*}\left(\boldsymbol{w} ; C^{F}\right)=\mathrm{E}\left(\bigwedge_{i=1}^{d} \frac{w_{i} T_{i+}^{\alpha}}{\mathrm{E}\left(T_{i+}^{\alpha}\right)}\right) \text { and } a^{*}\left(\boldsymbol{w} ; C^{F}\right)=\mathrm{E}\left(\bigvee_{i=1}^{d} \frac{w_{i} T_{i+}^{\alpha}}{\mathrm{E}\left(T_{i+}^{\alpha}\right)}\right) .
$$

Applying Theorem 3.1 to (2.3) and (2.5), we obtain the following corollary.

Corollary 3.1. Making the same assumptions as Theorem 3.1, the upper tail and extremal dependence parameters are given by

$$
\tau_{J}=\frac{\mathrm{E}\left(\bigwedge_{i=1}^{d}\left(\mathrm{E}\left(T_{i+}^{\alpha}\right)\right)^{-1} T_{i+}^{\alpha}\right)}{\mathrm{E}\left(\bigwedge_{i \in J}\left(\mathrm{E}\left(T_{i+}^{\alpha}\right)\right)^{-1} T_{i+}^{\alpha}\right)} \quad \text { for } \varnothing \neq J \subseteq N \quad \text { and } \quad \gamma=\frac{\mathrm{E}\left(\bigwedge_{i=1}^{d}\left(\mathrm{E}\left(T_{i+}^{\alpha}\right)\right)^{-1} T_{i+}^{\alpha}\right)}{\mathrm{E}\left(\bigvee_{i=1}^{d}\left(\mathrm{E}\left(T_{i+}^{\alpha}\right)\right)^{-1} T_{i+}^{\alpha}\right)}
$$

We illustrate our main results using multivariate elliptical distributions. Let $\boldsymbol{\Sigma}$ be a $d \times d$ positive semidefinite matrix, and let $\boldsymbol{U}=\left(U_{1}, \ldots, U_{m}\right)$ be uniformly distributed on the unit sphere (with respect to Euclidean distance) in $\mathbb{R}^{m}$. Consider the following stochastic representation:

$$
\left(X_{1}, \ldots, X_{d}\right)^{\top}=\left(\mu_{1}, \ldots, \mu_{d}\right)^{\top}+R \boldsymbol{A}\left(U_{1}, \ldots, U_{m}\right)^{\top},
$$

where $\boldsymbol{A}$ is a $d \times m$ matrix with $\boldsymbol{A A}^{\top}=\boldsymbol{\Sigma}$ and $R$ is a nonnegative random variable independent of $\boldsymbol{U}$. The distribution of $\left(X_{1}, \ldots, X_{d}\right)$ is known as an elliptical contoured distribution and is one of most widely used radially symmetric multivariate distributions. The examples of elliptical distributions include the multivariate normal, $t$-, and logistic distributions. We refer the reader to [6, Chapter 2] for details on properties of elliptical distributions.

Let $\left(T_{1}, \ldots, T_{d}\right)^{\top}=\boldsymbol{A}\left(U_{1}, \ldots, U_{m}\right)^{\top}$. Then we have

$$
\left(X_{1}, \ldots, X_{d}\right)=\left(\mu_{1}, \ldots, \mu_{d}\right)+R\left(T_{1}, \ldots, T_{d}\right),
$$

which is a scale mixture of $\left(T_{1}, \ldots, T_{d}\right)$. The random vector $\left(X_{1}, \ldots, X_{d}\right)$ is regularly varying if and only if $R$ has a regularly varying right tail [10], [21]. A formula for the bivariate tail dependence parameter has also been established in [10] and [21], and the upper tail dependence function of a multivariate elliptical distribution was explicitly derived in [13]. The multivariate upper tail dependence and exponent functions, and the upper tail and extremal dependence parameters for multivariate elliptical distributions are now given by Theorem 3.1 and Corollary 3.1. We discuss the bivariate case in detail. Let

$$
\boldsymbol{\Sigma}=\left[\begin{array}{ll}
\sigma_{11}^{2} & \sigma_{12} \\
\sigma_{12} & \sigma_{22}^{2}
\end{array}\right] \text { and } \boldsymbol{A}=\left[\begin{array}{cc}
\sigma_{11} & 0 \\
\sigma_{12} / \sigma_{11} & \sigma_{22} \sqrt{1-\rho^{2}}
\end{array}\right]
$$

where $\rho=\sigma_{12} / \sigma_{11} \sigma_{22}$. Thus,

$$
T_{1}=\sigma_{11} U_{1}, \quad T_{2}=\frac{\sigma_{12}}{\sigma_{11}} U_{1}+\sigma_{22} \sqrt{1-\rho^{2}} U_{2}
$$


Observe that, marginally, $R T_{1}$ and $R T_{2}$ have one-dimensional elliptical distributions, and $\sigma_{11}^{-1} R T_{1}$ and $\sigma_{22}^{-1} R T_{2}$ have the same distribution (see [6, Chapter 2]). Assume that $R$ has a regularly varying right tail with heavy tail index $\alpha>0$. Since $\left(X_{1}, X_{2}\right)$ and $\left(R T_{1}, R T_{2}\right)$ have the same tail dependence parameter, we have

$$
\tau_{1}=\lim _{t \rightarrow \infty} \operatorname{Pr}\left\{\sigma_{22}^{-1} R T_{2}>t \mid \sigma_{11}^{-1} R T_{1}>t\right\} .
$$

Obviously, $\left(T_{1}, T_{2}\right)$ has a bounded support; thus, it follows from Corollary 3.1 that

$$
\tau_{1}=\frac{\mathrm{E}\left(\sigma_{11}^{-1} T_{1+} \wedge \sigma_{22}^{-1} T_{2+}\right)^{\alpha}}{\mathrm{E}\left(\sigma_{11}^{-1} T_{1+}\right)^{\alpha}}=\frac{\mathrm{E}\left(U_{1+} \wedge\left(\rho U_{1}+\sqrt{1-\rho^{2}} U_{2}\right)_{+}\right)^{\alpha}}{\mathrm{E}\left(U_{1+}\right)^{\alpha}},
$$

where $U_{1+}:=U_{1} \vee 0$ and $\left(\rho U_{1}+\sqrt{1-\rho^{2}} U_{2}\right)_{+}:=\left(\rho U_{1}+\sqrt{1-\rho^{2}} U_{2}\right) \vee 0$.

If $\rho=1$ then, trivially, $\tau_{1}=1$. Suppose that $\rho<1$. Transferring to polar coordinates, we have

$$
U_{1}=\cos \Theta, \quad U_{2}=\sin \Theta, \quad \text { and } \quad \rho U_{1}+\sqrt{1-\rho^{2}} U_{2}=\sin \left(\Theta+\theta_{0}\right),
$$

where $\Theta$ is uniformly distributed on $[0,2 \pi]$ and $\theta_{0}=\tan ^{-1}\left(\rho / \sqrt{1-\rho^{2}}\right)$. Clearly,

$$
\mathrm{E}\left(U_{1+}^{\alpha}\right)=\frac{1}{2 \pi} \int_{-\pi / 2}^{\pi / 2} \cos ^{\alpha} \theta \mathrm{d} \theta=\frac{1}{\pi} \int_{0}^{\pi / 2} \cos ^{\alpha} \theta \mathrm{d} \theta=\frac{1}{\pi} \int_{0}^{1} \frac{u^{\alpha}}{\sqrt{1-u^{2}}} \mathrm{~d} u .
$$

To calculate the quantity in the numerator of (3.3), consider the boundary case when

$$
u_{1}=\rho u_{1}+\sqrt{1-\rho^{2}} u_{2},
$$

which leads to the solution $\theta_{1}=\tan ^{-1}\left(u_{2} / u_{1}\right)=\tan ^{-1}\left((1-\rho) / \sqrt{1-\rho^{2}}\right)$. That is,

$$
\begin{gathered}
\cos \theta \geq \sin \left(\theta+\theta_{0}\right) \geq 0 \quad \text { if }-\theta_{0} \leq \theta \leq \theta_{1}, \\
0 \leq \cos \theta \leq \sin \left(\theta+\theta_{0}\right) \quad \text { if } \theta_{1} \leq \theta \leq \pi / 2
\end{gathered}
$$

Simple trigonometric arguments show that $\theta_{0}+2 \theta_{1}=\pi / 2$. Thus,

$$
\begin{aligned}
\mathrm{E}\left(U_{1+} \wedge\left(\rho U_{1}+\sqrt{1-\rho^{2}} U_{2}\right)_{+}\right)^{\alpha} & =\frac{1}{2 \pi} \int_{-\theta_{0}}^{\theta_{1}} \sin ^{\alpha}\left(\theta+\theta_{0}\right) \mathrm{d} \theta+\frac{1}{2 \pi} \int_{\theta_{1}}^{\pi / 2} \cos ^{\alpha} \theta \mathrm{d} \theta \\
& =\frac{1}{2 \pi} \int_{0}^{\pi / 2-\theta_{1}} \sin ^{\alpha} \theta \mathrm{d} \theta+\frac{1}{2 \pi} \int_{\theta_{1}}^{\pi / 2} \cos ^{\alpha} \theta \mathrm{d} \theta
\end{aligned}
$$

Since $\cos \theta_{1}=\sqrt{1-\rho^{2}} / \sqrt{2-2 \rho}=((1+\rho) / 2)^{1 / 2}$, we obtain

$$
\mathrm{E}\left(U_{1+} \wedge\left(\rho U_{1}+\sqrt{1-\rho^{2}} U_{2}\right)_{+}\right)^{\alpha}=\frac{1}{\pi} \int_{0}^{((1+\rho) / 2)^{1 / 2}} \frac{u^{\alpha}}{\sqrt{1-u^{2}}} \mathrm{~d} u .
$$

Hence,

$$
\tau_{1}=\int_{0}^{((1+\rho) / 2)^{1 / 2}} \frac{u^{\alpha}}{\sqrt{1-u^{2}}} \mathrm{~d} u / \int_{0}^{1} \frac{u^{\alpha}}{\sqrt{1-u^{2}}} \mathrm{~d} u,
$$

which is identical to the one obtained in [10] and [21]. 
An intriguing issue is whether or not these tail dependence parameters are monotone in response to changes in the heavy tail index $\alpha$. It was demonstrated by the numerical results in [21] that the tail dependence parameter $\tau_{1}$ is decreasing in $\alpha$. We show that this is indeed the case using the ratio-of-moments expressions obtained in Corollary 3.1.

A nonnegative random variable $X$ is said to be smaller than a nonnegative random variable $Y$ in the hazard rate ordering, denoted by $X \leq_{\mathrm{hr}} Y$, if the hazard rate of $X$ is larger than that of $Y$. A detailed discussion on the hazard rate ordering can be found, for example, in Chapter 1 of [22], from which, the following result also holds.

Lemma 3.1. Let $X$ and $Y$ be nonnegative random variables. If $X \leq \mathrm{hr} Y$ then $\mathrm{E} X^{\alpha} / \mathrm{E} Y^{\alpha}$ is decreasing in $\alpha$.

Proof. Theorem 1.B.12 of [22] states that if $X \leq_{\mathrm{hr}} Y$ then

$$
\mathrm{E} g_{2}(X) \mathrm{E} g_{1}(Y) \leq \mathrm{E} g_{1}(X) \mathrm{E} g_{2}(Y)
$$

for all nonnegative real functions $g_{1}$ and $g_{2}$ satisfying the conditions that $g_{1}(\cdot)$ is increasing and $g_{2}(\cdot) / g_{1}(\cdot)$ is increasing. Consider the situation in which $\alpha_{1} \leq \alpha_{2}, g_{1}(x)=x^{\alpha_{1}}$, and $g_{2}(x)=x^{\alpha_{2}}$ for $x \geq 0$. Then (3.4) reduces to

$$
\mathrm{E} X^{\alpha_{2}} \mathrm{E} Y^{\alpha_{1}} \leq \mathrm{E} X^{\alpha_{1}} \mathrm{E} Y^{\alpha_{2}} .
$$

The monotonicity of $\mathrm{E} X^{\alpha} / \mathrm{E} Y^{\alpha}$ follows.

Proposition 3.1. Let $R T_{i}$ be the ith component of (1.1), where $T_{i}$ and $R$ satisfy the regularity conditions specified in Theorem 3.1. Suppose that $\mathrm{E}\left(T_{i+}^{\alpha}\right)=\mathrm{E}\left(T_{j+}^{\alpha}\right), i \neq j$.

1. If $\bigwedge_{i=1}^{d} T_{i+} \leq \mathrm{hr} \bigwedge_{i \in J} T_{i+}$ then $\tau_{J}$ is decreasing in $\alpha$.

2. If $\bigwedge_{i=1}^{d} T_{i+} \leq \mathrm{hr} \bigvee_{i=1}^{d} T_{i+}$ then $\gamma$ is decreasing in $\alpha$.

Proof. If $\mathrm{E}\left(T_{i+}^{\alpha}\right)=\mathrm{E}\left(T_{j+}^{\alpha}\right), i \neq j$, then, from Corollary 3.1,

$$
\tau_{J}=\frac{\mathrm{E}\left(\bigwedge_{i=1}^{d} T_{i+}\right)^{\alpha}}{\mathrm{E}\left(\bigwedge_{i \in J} T_{i+}\right)^{\alpha}} \quad \text { for all } \varnothing \neq J \subset N \quad \text { and } \quad \gamma=\frac{\mathrm{E}\left(\bigwedge_{i=1}^{d} T_{i+}\right)^{\alpha}}{\mathrm{E}\left(\bigvee_{i=1}^{d} T_{i+}\right)^{\alpha}}
$$

The monotone properties follow from Lemma 3.1.

Note that inequality (3.4) resembles the property of total positivity of order 2, and, in fact, Proposition 3.1 can be established directly by using the theory of total positivity.

To show that $\tau_{1}$ of a bivariate elliptical distribution is decreasing in $\alpha$, we need, by virtue of Lemma 3.1, to establish that $U_{1+} \geq_{\mathrm{hr}} U_{1+} \wedge\left(\rho U_{1}+\sqrt{1-\rho^{2}} U_{2}\right)_{+}$. From [22, Condition 1.B.3, p. 16], it is sufficient to show that

$$
\frac{\operatorname{Pr}\left\{U_{1+}>t\right\}}{\operatorname{Pr}\left\{U_{1+} \wedge\left(\rho U_{1}+\sqrt{1-\rho^{2}} U_{2}\right)_{+}>t\right\}} \text { is increasing in } t \in[0, s],
$$

where $s \leq 1$ is the right endpoint of the support of $U_{1+} \wedge\left(\rho U_{1}+\sqrt{1-\rho^{2}} U_{2}\right)_{+}$. Again, using the polar coordinate system, we have, for any $t \in[0, s]$,

$$
\begin{gathered}
\operatorname{Pr}\left\{U_{1+}>t\right\}=\frac{\cos ^{-1} t}{\pi}, \\
\operatorname{Pr}\left\{U_{1+} \wedge\left(\rho U_{1}+\sqrt{1-\rho^{2}} U_{2}\right)_{+}>t\right\}=\frac{\left(\cos ^{-1} t-\sin ^{-1} t\right)_{+}}{2 \pi} .
\end{gathered}
$$


It is easy to verify that $\frac{1}{2}\left(\cos ^{-1} t /\left(\cos ^{-1} t-\sin ^{-1} t\right)\right)$ is increasing in $t \in[0, s]$, and then (3.5) follows. Therefore, $\tau_{1}$ is decreasing in $\alpha$.

\section{Concluding remarks}

In this paper we have developed a general method based on multivariate regular variation to derive tail dependence parameters for heavy-tailed scale mixtures of multivariate distributions. Our method properly rescales the marginal distributions so that, asymptotically, the scaled random variables have the same tail marginal distributions; thus, the calculation of their tail dependence avoids taking the marginal transforms on the entire distribution. Our tail analysis leads to tractable expressions of tail dependence parameters which depend on joint moments of the random variables being mixed and the heavy tail index of the mixing random variable. Our method also establishes the link between multivariate tail dependence parameters and the theory of multivariate regular variation. The structural properties of multivariate tail dependence, such as monotonicity of tail dependence with respect to structural parameters of the distribution, have also been discussed for bivariate elliptical distributions. Yet, a general theory remains to be developed.

\section{Acknowledgement}

The authors would like to sincerely thank an anonymous referee for his/her insightful comments, which led to significant improvements in the results, contents, and presentation of this paper.

\section{References}

[1] Basrak, B., Davis, R. A. And Mikosch, T. (2002). A characterization of multivariate regular variation. Ann. Appl. Prob. 12, 908-920.

[2] Basrak, B., Davis, R. A. And Mikosch, T. (2002). Regular variation of GARCH processes. Stoch. Process. Appl. 99, 95-115.

[3] Bingham, N. H., Goldie, C. M. and Teugels, J. L. (1987). Regular Variation. Cambridge University Press.

[4] Buhl, C., Reich, C. AND Wegmann, P. (2002). Extremal dependence between return risk and liquidity risk: an analysis for the Swiss Market. Tech. Rep., Department of Finance, University of Basel.

[5] Embrechts, P., Lindskog, F. And McNeil, A. (2003). Modeling dependence with copulas and applications to risk management. In Handbook of Heavy Tailed Distributions in Finance, ed. S. Rachev, Elsevier, pp. 329-384.

[6] FAng, K. T., Kotz, S. AND NG, K. W. (1990). Symmetric Multivariate and Related Distributions. Chapman and Hall, London.

[7] Finkenstädt, B. And Rootzén, H. (2004). Extreme Values in Finance, Telecommunications, and the Environment. Chapman and Hall/CRC, New York.

[8] Frahm, G. (2006). On the extreme dependence coefficient of multivariate distributions. Statist. Prob. Lett. 76, 1470-1481.

[9] Hartmann, P., Straetmans, S., and de Vries, C. (2006). Banking system stability. A cross-Atlantic perspective. In The Risks of Financial Institutions, University of Chicago Press, pp. 133-192.

[10] Hult, H. ANd LindsKog, F. (2002). Multivariate extremes, aggregation and dependence in elliptical distributions. Adv. Appl. Prob. 34, 587-608.

[11] Joe, H. (1997). Multivariate Models and Dependence Concepts. Chapman and Hall, London.

[12] Joe, H., Li, H. And Nikoloulopoulos, A. K. (2009). Tail dependence functions and vine copulas. To appear in J. Multivariate Anal.

[13] KlüpPelberg, C., Kuhn, G. And Peng, L. (2008). Semi-parametric models for the multivariate tail dependence function-the asymptotically dependent. Scand. J. Statist. 35, 701-718.

[14] LI, H. (2008). Tail dependence comparison of survival Marshall-Olkin copulas. Methodology Comput. Appl. Prob. 10, 39-54.

[15] LI, H. (2009). Orthant tail dependence of multivariate extreme value distributions. J. Multivariate Anal. 100, 243-256.

[16] McNeil, A. J., Frey, R., Embrechts, P. (2005). Quantitative Risk Management. Princeton University Press. 
[17] Nelsen, R. B. (2006). An Introduction to Copulas, 2nd edn. Springer, New York.

[18] Nikoloulopoulos, A. K., Joe, H. And Li, H. (2009). Extreme value properties of multivariate $t$ copulas. Extremes 12, 129-148.

[19] ReSnick, S. I. (1987). Extreme Values, Regular Variation, and Point Processes. Springer, New York.

[20] Resnick, S. I. (2007). Heavy-Tail Phenomena. Springer, New York.

[21] Schmidt, R. (2002). Tail dependence for elliptically contoured distributions. Math. Meth. Operat. Res. 55, 301-327.

[22] Shaked, M. and Shanthikumar, J. G. (2007). Stochastic Orders. Springer, New York.

[23] Sklar, A. (1959). Fonctions de répartition à $n$ dimensions et leurs marges. Publ. Inst. Statist. Univ. Paris 8, 229-231. 